\title{
RAISONNEMENT SCIENTIFIQUE ET RÉSEAUX SOCIAUX DANS LA PHYSIQUE DE L'ENTRE-DEUX-GUERRES
}

Bruce R. Wheaton, The Tiger and the Shark. Empirical Roots of WaveParticle Dualism, with a Foreword by Thomas S. KuHN. Cambridge, Cambridge University Press, 1983. $16 \times 23,6,355 \mathrm{p}$.

Dominique PeSTRE, Physique et physiciens en France, 1918-1940. Paris, Ed. des Archives contemporaines, 1984. 13,5 $\times 21,356$ p., index (" Histoire des sciences et des techniques $\gg$ ).

Quelles sont les conditions sociales et organisationnelles, méthodologiques et épistémologiques susceptibles de bloquer ou, au contraire, de stimuler la recherche scientifique? On peut trouver des réponses à cette question dans les ouvrages récents de Bruce Wheaton et de Dominique Pestre sur l'histoire de la physique moderne. Dans The Tiger and the Shark : Empirical Roots of WaveParticle Dualism, Wheaton révèle l'importance de l'expérimentation dans le développement de la physique microscopique. Ses conclusions sont d'autant plus opportunes qu'elles corrigent une opinion couramment répandue parmi les historiens et les scientifiques eux-mêmes, opinion selon laquelle la physique moderne serait essentiellement un champ d'exploration théorique où le rôle de l'expérimentation au lieu de constituer un système autonome de découvertes, se réduirait à une confirmation des découvertes théoriques. Le livre de Wheaton est également précieux pour d'autres raisons. On y trouve en effet une multitude de renseignements détaillés fort utiles si l'on veut comparer les styles intellectuels et les contextes de recherche des scientifiques de nations différentes. Dans Physique et physiciens en France, 1918-1940, Dominique Pestre décrit, quant à lui, la production et la productivité des physiciens français pendant l'entre-deuxguerres. Selon lui, elles étaient faibles, pour ne pas dire nulles, en comparaison de celles des pays voisins. Il trouve à ce phénomène une série de causes intellectuelles et institutionnelles, sociales et psychologiques. Ce livre, par la quantité d'informations descriptives qu'il apporte, élargit notre connaissance d'une phase critique du développement scientifique en France.

Lorsqu'on lit en même temps les ouvrages de Wheaton et de Pestre, ceux-ci présentent un certain nombre de contradictions en ce qui concerne les moteurs de stimulation ou de blocage de la recherche. Certaines conditions que Pestre considère comme défavorables pour la science en France sont au contraire, aux yeux de Wheaton, des éléments stimulants, parfois même indispensables au

Revue de synthèse : IVe s. $\mathrm{N}^{\circ} 3$, juillet-septembre 1986. 
développement scientifique. Dans cette étude, je m'attacherai à analyser les contradictions que la lecture de ces deux livres met en lumière, puis j'essaierai de savoir ce que peuvent révéler ces paradoxes sur les dynamiques du processus de production du savoir. Je me pencherai en particulier sur l'étude du raisonnement scientifique et des réseaux sociaux des chercheurs, deux thèmes qui sont largement traités dans ces deux ouvrages.

Entre 1918 et 1939, la physique en France s'affaiblissait et était même, dans certaines branches, sur le point de s'effondrer. C'est du moins la conclusion la plus frappante du livre de Pestre. Selon lui, alors que le potentiel scientifique national n'avait fait que croître pendant la première moitié de la Troisième République, l'après-guerre a vu au contraire décliner de manière dramatique le nombre de personnes attirées par les carrières scientifiques, le montant des budgets des institutions et le niveau d'activité et de productivité des chercheurs. Cette situation, écrit-il, s'est quelque peu améliorée dans les années 1930, mais le niveau de la France n'en est pas moins resté faible dans la communauté internationale, particulièrement si on le compare avec celui de l'Angleterre, de l'Allemagne et des États-Unis.

Pestre a basé ce travail sur la productivité scientifique en France, sur deux types de documentations. Il a, d'une part, compté le nombre d'articles publiés par les scientifiques français dans plusieurs périodiques de physique français et internationaux. Ce nombre est bien inférieur à celui des productions des scientifiques des autres pays. Selon Pestre, leur travail était souvent de qualité très moyenne, et chose plus importante encore, il était souvent peu adapté aux préoccupations de la communauté internationale de physique. D'autre part, les manuels universitaires renforcent Pestre dans sa conviction que la physique française était dépassée. Il analyse le contenu des manuels d'électricité, d'électromagnétisme et de thermodynamique en les comparant à ceux des universités allemandes, anglaises et américaines. Au lieu d'une interprétation de la physique de leur époque, les manuels français dispensaient encore un enseignement du XIX siècle. Alors qu'à l'étranger les étudiants avaient à leur disposition des livres basés sur une vision synthétique et intégrée de la physique, les étudiants français devaient se contenter d'une représentation du savoir en physique à connotations extrêmement historiques, étroitement descriptive et empirique. L'enseignement français puisait alors l'essentiel de son contenu dans l'énumération séquentielle des données de cette discipline. Bien entendu, Pestre reconnaît que des chercheurs français tels que Langevin, Perrin, Cotton, Brillouin, Auger, Kastler, Maurice et Louis de Broglie ont contribué de manière significative à la science française et internationale. Mais il fait remarquer que ces scientifiques sont des exceptions et qu'ils ne reflètent ni le niveau scientifique ni l'efficacité du personnel scientifique de la nation. Selon lui, si leurs noms sont si fréquemment cités, c'est précisément qu'il y en a peu en France de leur calibre.

Pestre explique l'inadéquation de la physique française par les méthodes de recherche en faveur chez les scientifiques du pays, par la morphologie et les pratiques des établissements scientifiques et par la mentalité dominante du pays et de ses scientifiques. Selon lui, les techniques de recherche utilisées pendant l'entre-deux-guerres constituent une cause clé d'échec. Le raisonnement inductif 
dominait, à son avis, la vie de laboratoire, à l'exclusion de la pensée mathématique et déductive. Cela aboutissait à une pléthore d'observations et de descriptions, et à la collection minutieuse de faits - tout cela faisait oublier, à son avis, le besoin urgent de construire des programmes de recherche autour de l'analyse de paramètres soigneusement sélectionnés et de l'intégration des découvertes analytiques à des problématiques plus larges. Le savoir mathématique et les compétences étaient bien développés en France et la plupart des chercheurs en étaient les détenteurs (ceci grâce au système national de concours d'entrée aux Grandes Écoles et à la formation que l'on pouvait acquérir à l'École polytechnique et dans les établissements similaires), mais ils étaient rarement pleinement appliqués au travail expérimental. Il y avait un clivage entre l'excellence mathématique et le travail en physique : et quand les mathématiques étaient présentes de manière importante dans l'expérimentation, c'était généralement pour renforcer la pensée inductive plutôt que, selon Pestre, pour ouvrir de nouveaux champs d'investigation ou pour lier des résultats expérimentaux précis à des intérêts conceptuels plus larges. En somme, pour celui-ci, la physique était en France insuffisamment théorique dans son orientation. La théorie n'avait jamais été le point fort de la science française et cela a continué jusqu'aux années 1950. Mais au XXe siècle, écrit-il, le rôle du travail théorique pour le développement des connaissances se renforça, et l'incapacité ou le refus de nombreux physiciens à s'adapter à cette situation nouvelle provoqua une crise grave.

La politique de la nation, et l'organisation et le fonctionnement des établissements scientifiques affectèrent également l'orientation et le rendement de la physique. De 1918 à 1925, la France connut une inflation sans précédent : $600 \%$. Pendant cette période tous les établissements scientifiques importants du pays virent leur budget stagner. Cela eut, selon Pestre, un triple effet :

1) il y avait peu d'argent pour la recherche. Les scientifiques devaient parfois payer de leur poche pour pouvoir continuer leur travail. Ils étaient obligés de faire appel à l'aristocratie capitaliste pour financer de nouvelles fondations pour la recherche ;

2) pour des raisons budgétaires, le recrutement pour des postes scientifiques était minime. Les scientifiques potentiels se trouvaient ainsi attirés vers d'autres carrières ;

3) il en résulta une disproportion numérique entre les jeunes scientifiques peu nombreux et les plus âgés en nombre croissant. Cette situation créa une sorte de " gérontocratie ", selon le terme même de Pestre, qui étouffait l'introduction et le développement de nouvelles idées généralement associées au travail des jeunes chercheurs.

D'après Pestre, des critères institutionnels affaiblissaient également la physique en France. Des raisons budgétaires peut-être, des raisons de politique internationale très certainement, réduisirent ou interrompirent la participation des scientifiques français à la communauté scientifique mondiale pendant une bonne partie de l'entre-deux-guerres. Cet état de choses venait partiellement de la tentative des scientifiques français d'écarter les physiciens allemands qu'ils accusaient de trahison contre l'esprit scientifique pendant la guerre. Les Français continuaient à appliquer une politique d'ostracisme que les Anglais et les autres 
scientifiques d'Europe avaient abandonnée. Cela eut pour résultat d'isoler, non les Allemands, mais les Français eux-mêmes. Les scientifiques français assistèrent à un nombre relativement faible de conférences internationales entre 1918 et 1939, et Pestre indique que peu de laboratoires français ont invité des scientifiques étrangers pour de simples visites ou pour des projets de travail commun.

Enfin, Pestre voit dans la «mentalité française " une cause de retard scientifique. Selon lui, l'esprit scientifique français se caractérisait par le scepticisme, un positivisme naïf et une désaffection pour l'hypothèse et pour l'abstraction osée. Ces tendances s'opposaient à la créativité et à la spéculation imaginative. Empruntant à l'analyse de Jessie Pitts, Pestre insiste sur l'importance de la prouesse dans la pensée et le comportement français. Cette attitude, dit-il, est un obstacle : elle empêche les scientifiques de se réunir pour travailler ensemble afin de résoudre des problèmes complexes qui parfois nécessitent une division du travail scientifique. L'auteur stigmatise de la même façon le gaspillage et la dispersion de l'effort scientifique français à la fin du XIX' et au début du XX' siècle, gaspillage dû au clivage et aux conflits qui existaient entre les établissements et les scientifiques catholiques d'une part, républicains de l'autre. Cette situation s'est probablement prolongée, d'après Pestre, pendant l'entre-deux-guerres, privant ainsi la France d'une partie importante de ses talents scientifiques. S'appuyant enfin sur les travaux de Stanley Hoffman et de Michel Crozier, Pestre suggère que la communauté scientifique française était atteinte, comme le pays tout entier, d'une double maladie : celle de la " société bloquée " et du "phénomène bureaucratique ». Cette vision de la société française présente celle-ci comme une société marquée par le refus du risque et du changement. Cette situation, selon Pestre, créait un climat psychologique qui ne pouvait qu'inhiber le développement du potentiel de recherche du pays.

Malgré l'étendue et le caractère détaillé de cette étude, un doute subsiste sur plusieurs questions critiques. Tout d'abord, Pestre affirme que la physique française de l'entre-deux-guerres était en retard et improductive comparativement à la physique des autres pays, mais l'échantillon de la catégorie d'activités, et les sous-disciplines sur lesquels l'auteur s'appuie sont excessivement étroits et peu représentatifs de la physique dans son entier. La recherche de Pestre porte exclusivement sur la physique microscopique et, de façon plus étroite encore, sur la micro-physique théorique. Les propos de Pestre sur la physique microscopique théorique sont certainement convaincants, mais cela ne justifie pas qu'il tire, comme il le fait, de données si restreintes des conclusions générales sur la physique. Il n'y a, en effet, pas de raison logique pour que la physique microscopique fonctionne en France comme la physique macroscopique, ni la physique théorique comme la physique expérimentale. Il semble même peu raisonnable d'impliquer que la physique microscopique théorique reflète les autres branches de la physique au point de justifier une évaluation globale. En effet, pendant l'entre-deux-guerres, certaines sous-disciplines de la physique étaient, en France, à la fois productives et reconnues internationalement pour leur excellence. La France était un leader en physique des fluides. Le laboratoire de Henri Bénard à la Sorbonne était important, actif et productif, et en contact 
continu avec les chercheurs des autres pays. Campé de Fériet était également un chercheur reconnu dans ce domaine. Peut-être trop obnubilé par les faiblesses de la physique microscopique théorique, Pestre a omis de mentionner les performances de la science expérimentale en France. Cette lacune fondamentale du travail de Pestre conduit pour le moment à suspendre tout jugement sur le succès ou l'échec de la science française à cette époque. Il serait probablement instructif de formuler une réplique aux conclusions de Pestre, non pas en termes globaux et réductionnistes, mais plutôt en s'attachant aux productions des différents champs spécifiques de la physique.

En second lieu, il faut choisir avec beaucoup de prudence la catégorie d'indicateurs sur lesquels seront basées les mesures de performance. Les manuels d'étudiants, par exemple, sont une source de renseignements incontestablement intéressants et véritablement révélateurs sur la science. Mais la présentation du savoir faite à des étudiants dans un but pédagogique ne reflète pas nécessairement l'état de la discipline scientifique telle qu'elle est abordée dans les laboratoires et perçue par les scientifiques. La philosophie et les techniques d'enseignement sont en partie indépendantes de la vie de la discipline elle-même, et même si elles donnent certaines informations sur la pratique scientifique, elles informent peut-être mieux sur les conditions sociales, idéologiques et institutionnelles que sur les caractéristiques d'une discipline scientifique. De la même manière, si Pestre avait utilisé d'autres revues que Physical Review et le Journal de physique pour mesurer la productivité des physiciens français, ses conclusions auraient été certainement plus circonspectes et plus nuancées. Il est vrai que peu de scientifiques français ont publié leurs observations en physique microscopique théorique, mais il n'est pas moins vrai, preuves à l'appui, que les physiciens français ont abondamment publié, en particulier en physique des fluides, en physique mécanique, en acoustique, en optique... Il est intéressant de noter que dans son inventaire des publications des physiciens français dans Physical Review, Pestre reconnaît que ceux-ci étaient sur-représentés dans de nombreuses sous-disciplines en physique. Au lieu d'interpréter cette observation comme un indice de la fécondité des recherches françaises dans certaines disciplines, il préfère n'y voir qu'une preuve de la désaffectation des scientifiques étrangers pour cette revue. En somme, quand les Français ne publient pas, Pestre y voit un signe de faiblesse, et lorsqu'ils publient, il trouve cela insignifiant. En fin de compte, il reste encore à trouver des critères spécifiques et élaborés permettant de mesurer la production et la qualité de la recherche.

Troisièmement, il serait essentiel de poursuivre la recherche sur les préférences et les pratiques épistémologiques de la communauté des physiciens français. Selon Pestre, le raisonnement inductif dominait la physique de l'entre-deux-guerres, aussi bien dans la pédagogie que dans la recherche. Mais, comme nous l'avons dit plus haut, il convient d'aborder cette affirmation avec précaution. Si l'induction figurait, au moins sur un certain plan, dans les manuels universitaires, cela ne prouve pas qu'elle ait eu une place privilégiée dans les laboratoires. Pour Pestre, l'expérimentation et l'induction coïncident, et la seconde est dominante. Cette assertion prend implicitement racine dans la croyance que les opérations expérimentales sont exclusivement ou principalement liées à 
l'emploi de la pensée inductive ; et tout en faisant un plaidoyer pour le développement de la théorie qu'il considère comme le produit du raisonnement déductif, l'auteur critique l'emploi de l'induction. Mais cette perception de l'épistémologie est excessivement étroite et simple. Les processus de pensée des scientifiques embrassent plus de deux modes épistémologiques. Il n'est pas évident que l'expérimentation s'appuie exclusivement sur l'induction. À cause du concours d'entrée aux Grandes Écoles basé sur les mathématiques, et de l'importance de ces dernières dans les cursus des dites Grandes Écoles, la France a eu et continue à avoir un préjugé favorable pour la pensée déductive préjugé dont beaucoup de scientifiques français ont fait l'expérience, et dont ils ont souvent fait l'éloge. Il semble, de plus, que cette prédilection pour la déduction influence depuis longtemps les techniques et les analyses expérimentales françaises. Si l'expérimentation s'appuie sur certaines opérations inductives (particulièrement pendant l'observation et la collation des données d'observation), il n'en est pas moins vrai qu'en France le raisonnement déductif a généralement influencé les opérations expérimentales.

Le cadre conceptuel dans lequel les expériences étaient élaborées et les protocoles développés était étroitement conditionné par les algorithmes formels. Ici l'expérimentation dérivait des impératifs déductifs. En effet, on attendait des résultats expérimentaux qu'ils informent un appareillage conceptuel axiomatique hiérarchisé. Si l'induction avait été dominante en France, les résultats expérimentaux auraient mis en évidence des caractéristiques différentes : 1) Une très grande quantité de résultats auraient été obtenus, collationnés et traités, et ils auraient été systématiquement employés à construire une grille d'interprétations ou à différencier plusieurs interprétations possibles.

2) On aurait davantage tenu compte des anomalies expérimentales.

3) On aurait beaucoup plus mis l'accent sur une interprétation physique des événements et des propriétés physiques aussi bien que sur la construction d'une géométrie appropriée en opposition aux représentations formelles (ce point sera développé plus largement un peu plus loin). Mais aucun de ces traits ne caractérise l'expérimentation en France. Ce problème doit certainement être exploré plus en détail ; mais il semble d'ores et déjà que si l'induction opérait dans le processus d'expérimentation, celui-ci était généralement guidé et conditionné par des considérations déductives - situation qui existe encore actuellement en France.

Tandis que l'originalité et la force du livre de Pestre résident dans la somme d'informations provenant des archives et des publications ainsi que dans la qualité de ses descriptions, l'excellence du livre de Wheaton est due à sa synthèse des travaux de recherche menés dans de nombreuses sous-disciplines de la physique du début du $\mathrm{XX}^{*}$ siècle, dans des contextes très différents. Le titre de son livre, The Tiger and the Shark, lui fut suggéré par un article de J. J. Thomson où celui-ci. faisait référence à ces deux animaux. L'article de ce dernier exprime le malaise grandissant de nombreux physiciens des années 1920 devant la nécessité analytique de concevoir certaines catégories de la matière et de l'énergie dans l'optique de l'indéterminisme. Ce malaise était particulièrement aigu 
parmi les scientifiques britanniques qui avaient jusqu'alors favorisé une représentation mécaniste des événements physiques. Ce malaise se cristallisait autour d'une compréhension nouvelle de la nature de l'énergie de radiation, qui décrivait et expliquait les phénomènes électromagnétiques comme un dualisme particule-onde. Il était, en effet, difficile pour beaucoup de physiciens de saisir que l'énergie pouvait être simultanément corpusculaire et ondulatoire. Car ces deux propriétés avaient été jusque-là considérées comme incompatibles. Thomson comparait la relation paradoxale et complexe onde/particule qui caractérisait la nouvelle physique à la lutte d'un tigre et d'un requin, chacun ayant la suprématie dans son propre élément. L'histoire présentée par Wheaton est celle de l'évolution depuis les origines et de l'adhésion à la théorie dualiste de la lumière, qui a supplanté la théorie ondulatoire dans les années 1920, et qui tient une place importante dans la formulation de la théorie de la mécanique quantique.

En 1905, Einstein avait suggéré que la lumière avait des propriétés corpusculaires, particulièrement à hautes fréquences. D'après Wheaton, l'histoire de la théorie quantique de la lumière, et en particulier de sa dépendance par rapport à l'expérimentation, n'a pas attiré l'attention qu'elle méritait. Quant à la nature et au comportement de l'énergie de radiation, les physiciens reconnaissaient que les données empiriques mettaient en danger la théorie classique du rayonnement longtemps avant que celle-ci fût suffisamment modifiée pour s'accorder avec ces données. Une grande partie des informations qui ont contribué à faire accepter la théorie quantique de la lumière était centrée sur l'investigation des propriétés et actions des rayons $X$. C'était les résultats des recherches sur les rayons $X$ qui avaient conduit Louis de Broglie à proposer en 1922-1923 le dualisme onde/particule. De 1896 à 1907, la majorité des physiciens croyaient que les rayons $X$ étaient essentiellement différents de la lumière. Pour eux, les rayons $X$ étaient des pulsations plutôt que des ondes. Ils pensaient que, comme la lumière, les rayons $X$ se propageaient de façon sphérique à partir d'une source, mais ils ne les voyaient pas comme des oscillations périodiques. Ils les savaient temporellement discontinus, mais à ce stade de leur recherche, ils ne les croyaient pas localisés dans l'espace. Malgré les incertitudes, les ambiguïtés et les anomalies expérimentales considérables, Thomson en Grande-Bretagne et Sommerfeld en Allemagne se prononcèrent pour l'interprétation en termes de pulsations.

Si les ondes se répandent dans l'espace en occupant un volume de plus en plus grand, il n'en va pas de même des particules. De 1905 à 1912, les scientifiques travaillent sur ce paradoxe crucial de la diffusion des rayons X. De 1900 à 1910 , la recherche sur l'atome attire l'attention des physiciens sur l'existence de plusieurs événements subatomiques - les émissions alpha, beta et gamma. Après plusieurs années de recherche sur alpha et beta, Bragg se met à travailler sur le comportement des rayons $X$. Il note alors en soulignant son extrême importance le phénomène qui sera connu sous le nom de " paradoxe de la quantité ". Si les rayons $\mathrm{X}$ étaient ondulatoires, dit-il, ils induiraient une émission d'électrons par les atomes situés sur un plan infini. Si, en revanche, les rayons $X$ sont corpusculaires, leur effet sur les atomes est localisé dans l'espace. Selon Bragg, les résultats expérimentaux publiés jusque-là suggèrent que les rayons $\mathrm{X}$ ont des 
propriétés corpusculaires. Les travaux publiés par la suite sur les rayons $\mathrm{X}$ et les rayons gamma, cherchant à déterminer les sections efficaces lors des interactions entre des rayons $X$ et des atomes, ont révélé que ces sections efficaces n'étaient pas isotropes, mais comprises dans un cône. Pendant cette même période, les expériences de Dorn ont montré que l'énergie de l'électron déplacé par les rayons $\mathrm{X}$ est égale à l'énergie requise pour produire ces mêmes rayons $\mathrm{X}$. Leur énergie ne se disperse donc pas dans l'espace comme dans le cas des ondes. De p̈lus, les recherches de Dorn montraient que le comportement et la capacité de pénétration des rayons $X$ sont en corrélation avec leur fréquence. Vers 1911, Sommerfeld lui-même admettait que la fréquence constitue un des paramètres des rayons $X$, et que ceux-ci sont en quelque sorte associés aux particules. Cela le conduisit à envisager la possibilité de l'existence d'un continuum phénoménologique englobant des événements périodiques et a-périodiques.

De 1912 à 1921, la recherche sur les radiations s'est déplacée des rayons $X$ vers le problème de la lumière visible. En accord avec la seconde loi de Planck, les scientifiques ont généralement accepté que, même si les phénomènes d'émission affichent un comportement quantique, il y ait continuité dans l'absorption de l'énergie. Einstein fut le seul à penser que l'absorption fonctionne également de manière discontinue. Cette théorie photoélectrique d'Einstein sera confirmée par l'expérimentation. Cependant, et malgré la théorie d'Einstein, le rôle de la lumière dans l'effet photoélectrique n'était alors interprété que comme un mécanisme d'incitation. On pensait, en effet, que la lumière jouait exclusivement un rôle de " catalyse "; le transfert de l'énergie à l'électron était attribué à des interactions totalement étrangères à la lumière elle-même. Même après les expériences de Millikan révélant l'existence d'un transfert direct d'énergie entre la lumière et les électrons émis par des métaux exposés à la lumière, la plupart des physiciens refuseront de voir dans ce résultat la preuve de l'existence des quanta de lumière. Ils reconnaissaient cependant que le problème des composantes des radiations et de leur statut devenait de plus en plus complexe et, chose plus importante encore, de plus en plus crucial pour le développement de la physique microscopique. Exaspéré par ce problème, Bohr suggère alors que l'on gomme celui-ci en réduisant la portée de l'observation microscopique. Il propose que dorénavant on étudie l'énergie en calculant simplement les moyennes à partir d'un nombre important d'événements.

Tandis que les travaux sur les rayons $X$ et sur l'effet photoélectrique ont été nombreux en Angleterre et en Allemagne, la phase finale de la recherche sur la nature de l'énergie de radiation qui a abouti à la formulation du dualisme onde/particule s'est déroulée aux Etats-Unis et surtout en France. Et c'est spécialement en ce qui concerne la recherche en France que les résultats de Wheaton sont remarquables. Les travaux menés aux Etats-Unis commençaient alors à évoquer la possibilité d'une relation causale directe d'origine quantique entre l'absorption de la lumière et les phénomènes d'émission. Ces travaux faisaient revivre la possibilité d'attribuer à la lumière un caractère quantique, et ressuscitaient les problèmes touchant aux composantes corpusculaires et ondulatoires des radiations. Chose également importante, les travaux des frères de Broglie à Paris sur les paradoxes de l'énergie de radiation commençaient 
à ouvrir la voie à une compréhension synthétique de l'incompatibilité onde/particule.

Maurice de Broglie pensait que les rayons $\mathrm{X}$ et les rayons gamma faisaient partie intégrante du spectre électromagnétique. Pendant la Première Guerre mondiale, sa recherche portait sur l'absorption des rayons $X$. Il découvrit l'existence de trois niveaux d'absorption distincts. Pour Dauvillier, un collègue de Maurice de Broglie, les théories ondulatoires et corpusculaires sont difficiles à concilier, mais méritent néanmoins que l'on continue à travailler sur ce problème. Afin d'en apprendre davantage sur celui-ci - si ce n'est de le résoudre - Dauvillier utilisa les rayons $X$ pour induire des émissions d'électrons à partir des atomes en étudiant la gamme de leurs niveaux d'énergies. Il trouva une corrélation quantitative entre l'énergie utilisée pour induire l'émission d'électrons et celle de l'électron après son émission. Ainsi la radiation de fréquence " $N$ » expulse de l'élément « $R$ 》 des groupes d'électrons d'énergie « $K$ ». Une évidence s'est imposée à Maurice de Broglie et Dauvillier : chaque élément du spectre d'électrons correspond à un ensemble bien défini de gammes de vitesses dans le spectre corpusculaire.

Cette évidence était une raison, semblait-il, de penser, soit qu'une radiation était de nature corpusculaire, soit qu'elle devait correspondre à des points sur des surfaces d'ondes. Brillouin, en particulier, montra comment un électron décrivant une orbite périodique continue pouvait être décrit suivant des lois discontinues. Il émit l'idée de « point mobile » pour décrire le comportement des particules dans une onde. Il suggéra en particulier que son concept était probablement applicable à la lumière aussi bien qu'aux électrons. Mais c'est en fait Louis de Broglie qui fera une synthèse complète du dualisme onde/particule et qui indiquera sa validité pour toutes formes d'énergie de radiation, y compris la lumière. Détail intéressant, son point de départ ne fut pas l'énergie mais la matière. Suivant Louis de Broglie, à certains moments les propriétés corpusculaires prédominent, tandis qu'à d'autres ce sont les ondes. Pour expliquer les capacités de diffraction et d'interférence de la lumière, de Broglie dut remettre en question les lois classiques, ce qui le conduisit à établir de nouvelles lois sur les passages entre ces deux régimes. En définitive, le dualisme proposé par Louis de Broglie en 1922 et 1923 influera fortement sur le développement de la physique microscopique. Il sera au centre du travail de Schrödinger, et des expériences et des théories ultérieures sur la relation matière-énergie. En effet, tandis qu'Einstein faisait l'hypothèse sur la nature quantique de la lumière, la théorie synthétique de Louis de Broglie rendait crédible l'hypothèse d'Einstein et mettait en place les conditions favorables aux travaux expérimentaux et théoriques à venir.

Dans son histoire des racines empiriques du dualisme onde/particule, Wheaton fait la synthèse de la recherche des physiciens sur ce problème, ainsi que celle des résultats qu'ont obtenus les nombreux historiens qui ont écrit sur cet épisode historique de la science. Quoique tout à fait remarquable, cette synthèse aurait pu être faite à un niveau plus élevé. À noter un double problème : tout d'abord, la structure du récit de Wheaton souligne le caractère non linéaire de la recherche en physique. D'une certaine manière, ces récits se lisent comme un 
patchwork. Ses chapitres contiennent souvent une dizaine de sous-sections traitant de personnes particulières, de différentes catégories de phénomènes ou de techniques expérimentales. Mais si ce livre démontre implicitement par sa structure la non-linéarité du processus de recherche, il n'a rien à dire de particulier sur ce phénomène fondamental. Par exemple, il aurait fallu montrer précisément comment progresse la recherche sur les rayons $X$, les particules subatomiques et l'effet photo-électrique; puis, comment il lui arrive d'être peu à peu réduite et négligée. L'importance de ces différents domaines de la physique vient-elle de l'intérêt personnel de Wheaton pour la théorie onde/particule, ou bien chacun de ces domaines était-il vu par une partie importante de la communauté scientifique comme un terrain stratégique au moment où se faisait la recherche ? Dans le second cas, quels étaient les domaines susceptibles d'intéresser le plus de chercheurs? De plus, quelle était la perception de ceux qui travaillaient, par exemple, sur les rayons $\mathrm{X}$, comment organisaient-ils leur travail, quand leur recherche débouchait-elle sur un autre domaine? Pensaient-ils que leur spécialité continuerait d'offrir la clé susceptible de résoudre les questions critiques? Enfin, par quels mécanismes des domaines aussi hétérogènes que ceux que Wheaton décrit pouvaient-ils converger de manière à engendrer un nouveau concept révolutionnaire? Au début des années 1920, y avait-il un nombre important de chercheurs de la communauté internationale de physique sur le point de formuler la théorie sur le dualisme onde/particule ? Se pouvait-il que cette recherche ait été conduite en très peu de temps en Allemagne et en GrandeBretagne ? Ou au contraire le travail des physiciens français était-il si différent et inattendu qu'il constituait une classe à part ? En somme, Wheaton avait le matériel nécessaire à une réflexion fructueuse sur les aspects non linéaires de la recherche scientifique, et il est regrettable qu'il n'ait pas davantage développé ce thème.

Un second élément de synthèse aurait également été nécessaire. Les pratiques expérimentales diffèrent considérablement de la construction de la théorie scientifique. Le travail théorique consiste à sélectionner ou à élaborer une formule appropriée, à insérer dans cette formule des valeurs numériques utilisables ou des relations purement symboliques, à déterminer la façon la plus efficace d'agir sur la formule, et, enfin, à exécuter les opérations. L'expérimentation au contraire comporte une multitude de processus ambigus et transitoires : ajustements entre les problèmes scientifiques et les facilités expérimentales existantes, dessin et construction des instruments, établissement et exécution d'un protocole, ambiguités des mesures dues aux imperfections du système oculo-cérébral de l'homme, contamination phénoménologique, incertitude des anomalies phénoménologiques dans le traitement et l'analyse des données, etc. En effet, la pratique expérimentale recèle nombre d'ambiguités et d'obstacles qui font partie de la routine du travail. Wheaton était bien placé, en s'appuyant sur son étude copieuse et détaillée d'un certain nombre d'expérimentations spécifiques, pour donner un aperçu synthétique de la façon dont les physiciens expérimentaux ont perçu le processus de leur recherche, et ont parfois été amenés à conduire cette recherche dans un domaine manquant 
de prédécesseurs et de normes. La période de transition et de formation pendant laquelle des physiciens, qui avaient jusque-là travaillé en physique macroscopique, s'étaient tournés vers la physique microscopique, est certainement riche d'informations et de leçons. Comment, par exemple, les physiciens de cette époque se mesuraient-ils au problème de la validité des observations dans chacune de ces deux catégories? Quelles nouvelles opérations épistémologiques, lorsqu'il y en avait, étaient requises, et quelles nouvelles sources d'images mentales et physiques étaient évoquées pour saisir et illustrer ce qui était visuellement impossible à atteindre et qui souvent défiait l'expérience humaine ? Une analyse sociale et épistémologique des processus de production du savoir serait extrêmement utile, et une fois encore, les données de Wheaton renferment - à un niveau encore exploratoire il est vrai - les informations nécessaires à la réalisation de ce travail.

Wheaton, en effet, introduit une série de réflexions sur les tendances épistémologiques qui caractérisent les scientifiques anglais et allemands, réflexions qui, quoique évocatrices, éveillent chez le lecteur le besoin d'une information plus abondante et surtout plus précise. Wheaton reprend l'idée courante que les scientifiques britanniques appuyaient leur compréhension des phénomènes sur une interprétation mécanique. Ceci ne pouvait, selon Wheaton, que faire obstacle à leur travail de recherche sur les radiations. Mais la description de cette attitude reste beaucoup trop succincte. On aimerait savoir ce que Wheaton entend exactement par « une vision mécanique du monde ", et comment les scientifiques britanniques surmontaient cette tendance quand ils travaillaient sur les phénomènes quantiques. En effet, est-il exact, comme il l'implique, que la pensée fondée sur l'expérience sensorielle est incompatible avec la compréhension des phénomènes microscopiques ? Peut-être existe-t-il, en ce qui concerne le processus de compréhension, plus de convergence entre la physique microscopique et la physique macroscopique qu'on veut bien l'admettre en général. Peut-être que le monde microscopique devient intelligible au scientifique par une série de permutations et de transformations obliques au cours de l'expérimentation. Quoi qu'il en soit, si au tournant du siècle les Britanniques ont fonctionné suivant une tradition épistémologique particulière, qu'est devenue cette tradition lorsqu'elle s'est trouvée confrontée aux exigences de la physique microscopique ?

De la même façon, Wheaton indique de manière très intéressante que les physiciens allemands combinaient un fort penchant pour l'abstraction avec une sensibilité aux interprétations physiques des phénomènes. Il cite l'exemple de l'analyse des chercheurs allemands sur l'électromagnétisme. Pour eux, apparemment, l'électromagnétisme était essentiellement une abstraction formelle et mathématique tout en étant capable de manifestations matérielles. Cette ambiguïté posait quelques problèmes ; néanmoins, les scientifiques allemands réussirent à mener de front des recherches sur ces deux aspects de l'électromagnétisme. Mais ici également, on doit se demander ce qu'une telle attitude épistémologique signifie pour l'expérimentation et l'interprétation dans la recherche sur les radiations. Il est à noter que cette attitude ne permit pas aux physiciens allemands de devancer, comme on aurait pu le penser, les scientifiques français dans le développement de la théorie du dualisme 
onde/particule. Si l'analyse de Wheaton sur les penchants épistémologiques des différentes communautés scientifiques nationales est intéressante, elle reste incomplète et aurait certainement mérité d'être davantage élaborée et illustrée.

Enfin l'étude de Wheaton soulève des questions sur la dynamique qui sous-tend le développement du savoir scientifique. Les idées scientifiques nouvelles sont-elles un produit de l'irrationnel, en l'occurrence de l'émergence de nouvelles alliances sociales, ou sont-elles au contraire le produit d'une réflexion attentive liée à l'emploi de mesures, au souci des conditions limites, et à l'esprit critique ? L'analyse de Wheaton souligne la primauté de l'expérimentation systématique, du désintéressement individuel, et de l'usage de l'esprit critique dans la formulation de la théorie du dualisme. De plus, selon lui, la théorie a émergé peu à peu et non dans un éclat révolutionnaire, et sa formulation était indépendante des intérêts sociaux. Mais le livre de Wheaton contient une introduction de Kuhn qui, dans The Structure of Scientific Revolutions, émet l'idée que le savoir scientifique suit une dynamique discontinue, qu'il se développe en réponse à la pression d'intérêts de groupes, et qu'il prend la forme de cataclysmes conceptuels. Pour certains, les remarques de Kuhn confèrent une certaine légitimité aux analyses de Wheaton. Pourtant une lecture attentive de ces dernières met en évidence une certaine divergence entre elles et la position de Kuhn sur la façon dont se développe la science.

\section{I. - CONFRONTATIONS}

Wheaton et Pestre mettent tous deux l'accent sur l'importance du mélange mathématiques et expérimentation, abstrait et concret, mélange qui sous-tend les processus de pensée des physiciens et qui joue un rôle important dans le succès de leur recherche. Comme on l'a dit plus haut, Pestre considère qu'un excès de dépendance vis-à-vis de l'induction et de l'expérimentation, et un manque d'attention pour la convergence mathématiques/expérimentation sont des défauts profonds de la physique de l'entre-deux-guerres en France. Ils ont, à son avis, empêché la France de produire des théories. Pestre admet en même temps, cependant, que la quantité et la complexité des mathématiques utilisées en France étaient considérablement plus grandes qu'en Allemagne. Les observations de Wheaton conduisent celui-ci à peu près aux mêmes conclusions. Si c'est une description précise de la présence des mathématiques dans la recherche en France et en Allemagne, que reste-t-il de l'assertion de Pestre suivant laquelle le salut de la physique théorique en France aurait pu venir d'une utilisation plus grande des mathématiques, alors même que les Allemands les utilisaient moins, et sous des formes moins avancées, ce qui ne les empêcha pas de générer plus de théories? Le dilemme mis en évidence par le travail de Pestre est fondamental, mais la solution est sûrement plus complexe et nuancée qu'il l'indique. Les mathématiques jouaient en France au moins quatre rôles importants dans le processus de recherche : 
1) Elles étaient utilisées pour résoudre des problèmes numériques.

2) Elles servaient à élaborer et à clarifier des relations symboliques.

3) La déduction, corollaire épistémologique des mathématiques, neutralisait le potentiel contenu dans le raisonnement inductif.

4) Mathématiques et déduction prises ensemble offraient un cadre rigide qui rendait difficile la perception de la structure des classes de phénomènes, et les relations et les échanges entre ces classes.

Dans chacun de ces quatre rôles, les mathématiques et leur support déductif tendaient à constituer le mode dominant de perception, de construction et de déconstruction du monde physique. Bien entendu, la quantité et la sorte de mathématiques utilisées dans la recherche sont importantes, mais la manière dont celles-ci sont employées dans le but d'élucider des phénomènes constitue un paramètre encore plus fondamental. En France, l'expérimentation et la pensée inductive qui lui est associée étaient conditionnées par des impératifs mathématiques déductifs. Les mathématiques n'étaient pas confinées au statut d'outil analytique, elles étaient au contraire un point référentiel de base. En effet, beaucoup de physiciens français se tournaient vers les mathématiques en tant que système de représentation, au détriment d'autres modes de représentation comme les représentations iconiques, géométriques, mécaniques, linguistiques, etc. En Allemagne, la place des mathématiques dans la production du savoir était complètement différente. Cependant, la différence réside plutôt dans le rôle qu'elles ont joué que dans la quantité d'utilisation que l'on en faisait. Dans ses réflexions sur la compréhension de l'électromagnétisme par les physiciens allemands, Wheaton précise les fonctions des mathématiques dans la physique allemande. Les scientifiques allemands limitaient le plus souvent leur dépendance vis-à-vis des mathématiques. En effet, ils ne les considéraient et ne les utilisaient que comme un outil d'analyse et d'investigation parmi beaucoup d'autres. Pour eux, elles étaient un simple « opérateur » et n'étaient pas investies d'autres vertus. Cet usage restreint et prudent des mathématiques était lié à une seconde pratique de base. Les paramètres les plus abstraits de la nature étaient vus eux-mêmes comme possesseurs de manifestations concrètes. Dans le cas de l'électromagnétisme, alors que certaines propriétés ne pouvaient s'exprimer directement que par les mathématiques, les scientifiques allemands affirmaient que la représentation mathématique convergeait avec la manifestation matérielle. Ici les propriétés matérielles n'étaient vues que comme un reflet partiel de l'ontologie physique. En effet, en Allemagne, les représentations mathématiques servaient à construire ou à affiner les interprétations physiques des phénomènes. En France, en revanche, les scientifiques se méfiaient des interprétations physiques. 


\section{II. - QUELLES FORMES DE RÉSEAUX SOCIAUX ENCOURAGENT - OU AU CONTRAIRE INHIBENT - L'INNOVATION DANS LA PRODUCTION DU SAVOIR ?}

Wheaton et Pestre proposent deux réponses contradictoires à cette question. Pour Pestre, l'isolement des physiciens français pendant l'entre-deux-guerres les a empêchés de se poser les questions adéquates et d'acquérir de nouvelles techniques de recherche. Wheaton, quant à lui, souligne deux exemples dans lesquels les scientifiques travaillant à l'écart des hauts lieux de la recherche, ont engendré des concepts et des données d'une grande importance. Quand Bragg a présenté le début de sa synthèse qui traite simultanément des paradoxes de quantité et de qualité, il travaillait seul en Australie, loin des centres d'activité allemands ou britanniques. L'isolement physique et la distance intellectuelle où il se trouvait lui ont apporté, semble-t-il, un certain détachement vis-à-vis de la controverse qui animait alors ces centres d'activité, et lui ont ainsi permis de chercher une compréhension large et non partisane des ambiguïtés du problème des radiations. Wheaton insiste également sur l'isolement des frères de Broglie - qui étaient indépendants, à une époque d'institutionalisation de plus en plus intense, et qui travaillaient en France alors que les recherches précédentes en ce domaine avaient, pour les plus importantes au moins, été conduites dans d'autres pays. Les mesures inventives et les conclusions pénétrantes de Maurice de Broglie, les théories imaginatives et synthétiques de Louis de Broglie ont vu le jour en marge du pouvoir social et des analyses à la mode. Donc si Wheaton a raison, la production du savoir implique qu'une partie au moins des recherches se fasse dans un isolement relatif, pour échapper aux contraintes d'un conformisme et d'une soumission excessive aux préjugés en vogue.

En somme, malgré leurs différences, les livres de Wheaton et de Pestre apportent, en particulier quand on les lit en parallèle, une contribution importante à l'histoire de la physique du Xx' siècle. Le fait qu'ils arrivent à des conclusions différentes témoigne en quelque sorte des progrès constants dans l'étude historique des sciences. Même en travaillant sur la base de questions très différentes et avec des techniques également différentes, ces deux historiens convergent finalement sur l'identification des questions clés ; et si leurs réponses ne sont pas encore complètes, ils ont néanmoins parcouru une distance importante sur le chemin de la compréhension du contexte social de la recherche scientifique.

* Texte traduit de l'anglais par Michelle de LAUnAY. 